\title{
简并三能级原子在绝热极限下的量子 非破坏测量*
}

\author{
刘夏姬 \\ (东北师范大学理论物理研究所, 长春 130024)
}

\section{关键词量子非破坏测量 绝热近似 简并三能级原子}

由于微弱信号的测量, 如量子宇宙学中引力波的探测给实际测量提出了新的要求, Braginsky ${ }^{[1]}$ 等人提出了量子非破坏测量的概念 (quantum-nondemolition measurement 简称 QND 测量) 即第一次测量对被测物体的待测力学量没有扰动, 再次进行观测能重复第一次的测 量结果的测量称为量子非破坏测量. 正是由于它的这个性质引起了很多实验和理论物理学家的关 注 ${ }^{[2 \sim 6]}$. 本文通过研究一个简并的三能级原子与光场的相互作用, 由精确解计算给出在绝热 极限下可实现此类原子的动量非破坏测量.

\section{1 模型及其精确解}

我们考虑一个简并的三能级原子在一个与原子波长尺度可比拟的环形腔场中, 质心沿腔 轴 $X$ 方向运动, 原子和腔场相互作用的整体哈密顿量在旋转波近似下为:

$$
\begin{gathered}
\hat{H}=\frac{\hat{P}^{2}}{2 M}+h \omega_{0}\left(\left|e_{1}><e_{1}\right|+\left|e_{2}><e_{2}\right|\right)+h \omega a^{+} a+ \\
h g\left[\mathrm{e}^{-i q \hat{x}} a^{+}\left(\left|g><e_{1}\right|+\mu\left|g><e_{2}\right|\right)+\mathrm{e}^{i q \hat{x}} a\left(\left|e_{1}><g\right|+\mu\left|e_{2}><g\right|\right)\right],
\end{gathered}
$$

式中 $\hat{x}, \hat{P}$ 是原子质心位置和动量算符. $\left|e_{1}\right\rangle,\left|e_{2}\right\rangle$ 是两个简并的激发态, $|g\rangle$ 是基态. $h \omega_{0}$ 是 $\mid g>$ 和 $\left|e_{1}>,\right| e_{2}>$ 的能级差, $a^{+} a$ 是频率为 $\omega(\omega=c q)$ 的单模光场的产生, 湮灭算符, $g$ 是原子与 光场的耦合常数. $q$ 是行波光场的波数.

引人么正变换 ${ }^{[\text {: }}$ :

$$
W_{t}=\mathrm{e}^{\left.-i \omega t\left(a^{+} a+\left|e_{1}\right\rangle<e_{i}|+| e_{2}><e_{y}\right\rangle\right)+i q \hat{x}\left(\left|e_{1}><e_{i}\right|+\mid e_{2}><e_{j}\right)} .
$$

设 $|\psi(l)>=W| \widetilde{\psi}(t)>$ 是 $\hat{H}$ 支配 Schrodinger 方程的解, 则支配 $\mid \widetilde{\psi}(t)>$ 演化的哈密顿量是

$$
\begin{gathered}
\tilde{H}=\frac{\hat{P}^{2}}{2 M}-\hbar \Delta(\hat{P})\left(\left|e_{1}><e_{1}\right|+\left|e_{2}><e_{2}\right|\right)+ \\
h g\left[a^{+}\left(\left|g><e_{1}\right|+\mu\left|g><e_{2}\right|\right)+a\left(\left|e_{1}><g\right|+\mu\left|e_{2}><g\right|\right)\right],
\end{gathered}
$$

1994-11-21 收稿, 1995-04-12 收修改稿

*团家们然科黄基金资䏚项们 
式中 $\Delta(\hat{p})=\omega-\left(\omega_{0}+\frac{q \hat{p}}{M}+\frac{\hbar q^{2}}{2 M}\right), q \hat{p} / M$ 是 Doppler 频移.

求解 (3) 式的哈密顿量得到基本征值

$$
\begin{aligned}
E_{0} & =\frac{p^{2}}{2 M}(\text { 基态 }), \\
E_{n} & =\frac{p^{2}}{2 M}-\hbar \Delta(p), \\
E_{n}(+) & =\frac{p^{2}}{2 M}-\frac{\hbar}{2} \Delta(p)+\hbar W_{n}, \\
E_{n}(-) & =\frac{p^{2}}{2 M}-\frac{\hbar}{2} \Delta(p)-\hbar W_{n}
\end{aligned}
$$

和相应的本征函数:

$$
\begin{gathered}
|0, g>\otimes| P>(\text { 基态 }), \\
\left|\varphi_{n}>=\left(\sin \alpha\left|e_{1}, n>-\cos \alpha\right| e_{2}, n>\right) \otimes\right| p>, \\
\left|\varphi_{n}(+)>=\left[\sin \frac{\Theta_{n}}{2}\left(\cos \alpha\left|n, e_{1}>+\sin \alpha\right| n, e_{2}>\right)+\cos \frac{\Theta_{n}}{2} \mid n+1, g>\right] \otimes\right| p>, \\
\left|\varphi_{n}(-)>=\left[-\cos \frac{\Theta_{n}}{2}\left(\cos \alpha\left|n, e_{1}>+\sin \alpha\right| n, e_{2}>\right)+\sin \frac{\Theta_{n}}{2} \mid n+1, g>\right] \otimes\right| p>,
\end{gathered}
$$

其中

$$
\begin{array}{cc}
W_{n}=\sqrt{2 g^{2}(n+1)+\frac{\Delta^{2}(p)}{4}}, & \sin \alpha=\frac{\mu}{\sqrt{2}}, \\
\operatorname{tg} \Theta_{n}(p)=\frac{2 g \sqrt{2(n+1)}}{\Delta(p)}, & \cos \alpha=\frac{1}{\sqrt{2}} .
\end{array}
$$

$\mid p>$ 是原子质心动量的本征态, $\mid n>$ 是光场粒子数算符 $a^{+} a$ 的本征态. 由此, 我们有模型 的一个精确解:

$$
\begin{aligned}
\mid \widetilde{\psi}(t)>= & \int \mathrm{d} p\left\{\sum _ { n } \left[A_{n}(p) \mathrm{e}^{-\frac{i}{h} E_{p, n}(+) t}\left|\varphi_{n}(+)>+B_{n}(P) \mathrm{e}^{-\frac{i}{h} E_{p r n}(-) t}\right| \varphi_{n}(-)>+\right.\right. \\
& \left.\left.C_{n}(p) \mathrm{e}^{-\frac{i}{h} E_{n, n} t} \mid \varphi_{n}>\right]+D_{0}(p) \mathrm{e}^{-\frac{i}{h} E_{0} t} \mid 0, g>\right\} \otimes \mid p>,
\end{aligned}
$$

式中 $A_{n}(p), B_{n}(p), C_{n}(p), D_{0}(p)$ 是由初始条件确定的常数.

\section{2 绝热近似及其量子非破坏测量}

考虑到弱耦合情况, 当光场很弱且失谐很大 $\delta=\omega-\omega_{0}$, 亦即 $\delta /[g \sqrt{n+1}] \gg 1$. 我们有 一阶近似: 


$$
\begin{gathered}
W_{n}=\sqrt{2 g^{2}(n+1)+\frac{\Delta^{2}(p)}{4}} \simeq \frac{\Delta(p)}{2}+\frac{2 g^{2}}{\Delta(p)}(n+1), \\
\operatorname{tg} \Theta_{n}=\frac{2 g \sqrt{2(n+1)}}{\Delta(p)} \simeq 0 \quad \Theta_{n} \simeq 0,
\end{gathered}
$$

此时

$$
\begin{gathered}
E_{n}=\frac{p^{2}}{2 M}-h \Delta(p), E_{n}(+) \simeq \frac{p^{2}}{2 M}+h \Omega(P)(n+1), \\
E_{n} \simeq \frac{p^{2}}{2 M}-h \Delta(p), \\
E_{n}(+) \simeq \frac{p^{2}}{2 M}+h \Omega(p)(n+1), \\
E_{n}(-) \simeq \frac{p^{2}}{2 M}-h \Delta(p)-h \Omega(p)(n+1), \\
\left|\varphi_{n}>\simeq\left(\sin \alpha\left|n, e_{1}>-\cos \alpha>\alpha\right| n, e_{2}>\right) \otimes\right| p> \\
\left|\varphi_{n}(+)>\simeq\right| n+1, g>\otimes \mid p>, \\
\left.\left|\varphi_{n}(-) \simeq\right| g>\alpha\left|n, e_{1}>+\sin \alpha\right| n, e_{2}\right) \otimes \mid p>,
\end{gathered}
$$

式中 $\Omega(p)=\frac{g^{2}}{\Delta(p)}$, 从而精确解的波函数 (7) 取其绝热极限为

$$
\begin{gathered}
\mid \Psi(t)>=\int \mathrm{d} p-\sum_{n}\left[A_{n}(p) \mathrm{e}^{-\frac{i}{h} t\left|l_{2 M}^{2}+\Omega(p)(n+1)\right|} \mid n+1, g>+\right. \\
B_{n}(p) \mathrm{e}^{-\frac{i}{h} t\left|\frac{i}{2 M}-h(p)-\Omega(p)(n+1)\right|}\left(\cos \alpha\left|n, e_{1}>\right| \sin \alpha \mid n, e_{2}>\right) \\
\left.C_{n}(p) \mathrm{e}^{-\frac{i}{h} t \mid \frac{p^{2}}{2 M}-h \Delta(p) !}\left(\sin \alpha\left|n, e_{1}>-\cos \alpha\right| n, e_{2}>\right)+D_{0}(p) \mathrm{e}^{-\frac{i p^{2} t}{2 h M}} \mid 0, g>\right] \otimes \mid p>.
\end{gathered}
$$

上述近似中, 振幅中取零级近似, 位相中取一级近似 ${ }^{[n}$. 在这种近似下, 我们有绝热近似 哈密顿:

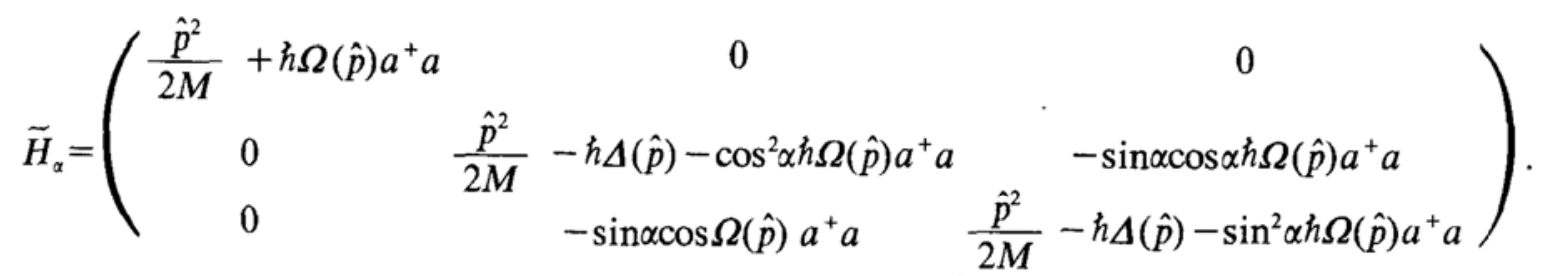

对应于原表象下绝热近似的哈密顿量为: 


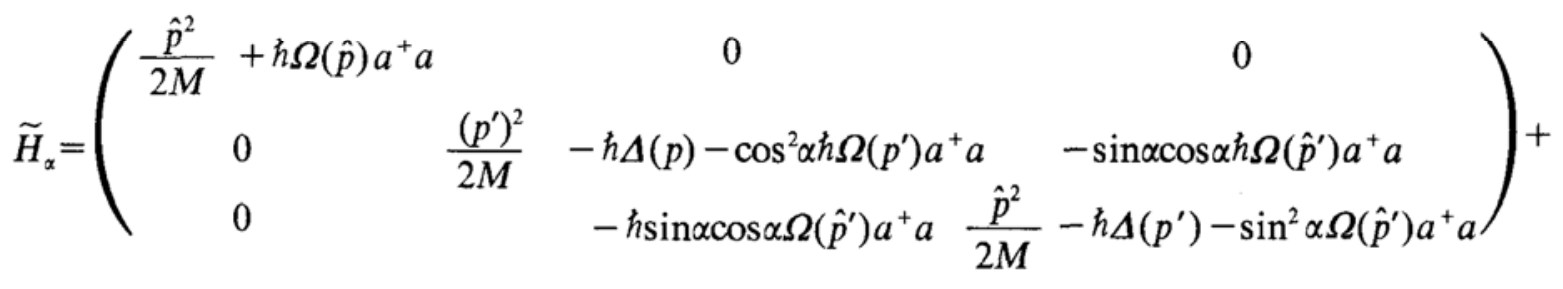

$$
\begin{aligned}
& h \omega\left(\begin{array}{ccc}
a^{+} a & 0 & 0 \\
0 & a^{+} a+1 & 0 \\
0 & 0 & a^{+} a+1
\end{array}\right) . \\
& \hat{p}^{\prime}=\hat{p}-q h
\end{aligned}
$$

由 $\hat{H}_{\alpha}$ 的表达式我们可以看出 $\left[\hat{H}_{\alpha}, \hat{P}\right]=0$, 由文献 [1] 我们知道这种情况下我们对光场力学 量 $\hat{Y}=\left(a^{+}+a\right) / 2^{[6]}$ 进行测量不影响原子的动量 $\hat{P}$, 所以此时通过 $\hat{Y}$ 与其关联确定 $\hat{P}$ 的测量是量 子非破坏测量.

由 (1) 式的哈密顿量我们知道 $[\hat{H}, \hat{P}] \neq 0$, 所以不作绝热近似条件下, 由光场力学量确定原 子动量的测量是量子破坏性测量, 绝热近似是进行量子非破坏测量的条件.

致谢 本文作者深深感谢导师孙昌丵教授的精心指导和耐心帮助.

\section{参考文献}

1 Braginsky V, Khallili F. Quantum Measurement. Cambridge: Great Britain at the University Press, 1992

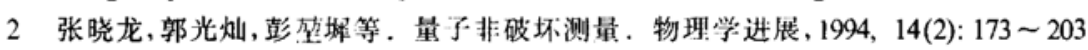

3 Grangier P. Quantum nondemolition measument experiment. Phys Rev, 1992, 219: 121

4 Marte M, Zoller P. Quantum nondemolition measument of transverse atomic position in kapitza-dirac atomic beam scattering. Appl Phys, 1992, B54: $477 \sim 485$

5 Sleator T, Wilkens M. Quantum-nondemolition measurement of atomic momentum. Phys Rev, 1993, A48: $3286 \sim 3289$

6 Storey P, Collett M, Walls D. Atomic-position resolution by quadrature-field measurement. Phys Rev, 1993, A47: 405

7 Sun C P(孙昌璜), Zhang L Z. Test of quantum adiabatic approximation via exactly-solvable pynamics of high-spin precession. Physica Scripta, 1995, 51: 16 18 